\title{
Chemical Biology of DNA Polymerases: From Selectivity to New Functions
}

\author{
Andreas Marx*, Daniel Summerer, Katharina B. M. Sauter, Christian Gloeckner, Nicolas Z. Rudinger \\ Department of Chemistry, Universitätsstrasse 10, D-78457 Konstanz, Germany
}

\begin{abstract}
DNA polymerases are involved in all DNA synthesis occurring in nature. Furthermore, DNA polymerases are the workhorses in numerous important molecular biological core technologies like the ubiquitous polymerase chain reaction (PCR), cDNA cloning, genome sequencing and nucleic acids based diagnostics.

In order to identify DNA polymerase mutants with altered properties, we set up an efficient highthroughput setup to rapidly screen libraries of DNA polymerase mutants in automated parallel fashion and identified entities with significantly increased selectivity. Furthermore, our results indicate a rational to generally increase DNA polymerase selectivity as we demonstrate for several enzymes from different DNA polymerase families.

Additionally, we show that the generation of a new DNA polymerase function is achievable through iterative screening of small libraries of DNA polymerase derived by randomization of the respective genes. We demonstrate that the identified mutants find immediate applications and provide the basis for the development of new means for diagnostic technologies.
\end{abstract}

\section{INTRODUCTION}

DNA polymerase activity is central in numerous biotechnological applications. However, the imperfect nature of DNA polymerase Watson-Crick fidelity under unnatural conditions employed in several techniques restricts their application or demands tedious optimization. Thus, increasing the fidelity of DNA polymerase function is an attractive goal. Alteration of DNA polymerase function by directed enzyme evolution has been shown to be very promising in the past.' We describe an efficient high-throughput setup to rapidly screen libraries of DNA polymerase mutants in automated parallel fashion. The readout is exclusively based on enzyme activity through fluorescence detection of the reaction product. Additionally, we describe successful applications of the approach for the discovery of DNA polymerases with increased fidelity and broadened substrate spectra.

\section{RESULTS AND DISCUSSION}

DNA polymerases with increased selectivity. A prime target for the design of altered DNA polymerase function is its Watson-Crick fidelity of DNA synthesis. Progress along this line generates valuable tools for a multitude of biological applications like PCR, sequencing protocols, mutagenesis techniques or genotyping. Recently, motif $\mathrm{C}$ was suggested to be involved in a common mechanism of A- and B-family DNA polymerases for mismatch recognition in the primer template via indirect minor groove $\mathrm{H}$-bonding. ${ }^{2}$ In A-family DNA polymerases the crucial amino acids are within a $\beta$-strand-turn- $\beta$-strand structure that harbors acidic side chains binding catalytically essential magnesium ions. Motif $\mathrm{C}$ is highly conserved among A-family DNA polymerases like KF but also among sequence families $\mathrm{B}, \mathrm{RT}, \mathrm{X}$, single subunit RNA polymerases and the lesion bypass DNA polymerases of the Y-family. ${ }^{3}$ Thus, motif $\mathrm{C}$ appeared to us as a promising target for directed reengineering through focused randomization and subsequent screening.

To reengineer motif $\mathrm{C}$ we constructed a library of $1316 \mathrm{KF}$ mutants randomized at the consensus residues comprising the amino acids Q879, V880, and H881. ${ }^{4}$ Protein expression was conducted in 96 well plates. Enzymes were screened directly after lysis and dilution without requiring further purification steps. DNA polymerase activities were monitored by SYBRgreen I mediated quantification of synthesized double stranded DNA after reaction termination. This setup links enzyme activity to a signal without requiring artificial substrates during reaction that might interfere with the enzymatic reaction. We comparatively assayed single clones in 384-well plates for their differential capability to extend primer templates harboring either a primer T-template A match or a TG mismatch at the primer 3 '-terminus. An automated pipetting device allowed fidelity evaluation of all library members in a few hours. As measurements of extension fidelity, ratios of measured fluorescence in case of match- versus mismatch-extension were calculated. Screening revealed considerable mutability of the targeted sequence QVH. Albeit this motif is highly conserved among several DNA and RNA polymerase families; $47 \%$ of the mutants exhibit measurable activity. We selected several clones with enhanced fidelity. We chose the three most selective mutants PLQ, LVG and LVL for further characterization and assayed the identified mutants in radiometric primer extensions. Measurements 
revealed that KF wt is capable of extending almost all mismatches under the chosen conditions albeit to varying extent. In contrast, all three selected mutants show markedly decreased efficiency of mismatch extension. Particularly, mutant LVL extends mismatched primer termini with only up to two nucleotides, whereas full-length mismatch extension is undetectable in most cases.

In addition, we could show that hydrophobic substitutions as found in the screen can be transferred to Taq DNA polymerase (family A DNA polymerase) ${ }^{4,5}$ as well as to Pfu DNA polymerase (family B DNA polymerase). ${ }^{6}$

DNA polymerases with broadened substrate spectra. The specific detection of RNA through reverse transcription and subsequent cDNA amplification is fundamental for many applications in diagnosis e.g. pathogene detection or gene expression analysis. ${ }^{7}$ In order to PCR amplify RNA usually two enzymes have to be employed: a reverse transcriptase (RT) that synthesizes the complementary DNA strand and a thermostable DNA polymerase for PCR amplification. However, the first step is prone to failures due to formation of stable RNA secondary structures and the diminished thermostability of the RT. Thus, we became interested in the development of a thermostable DNA polymerase that is competent to reverse transcribe RNA and subsequent PCR amplification without requiring $\mathrm{Mn}^{2+8}$. Here we show that the generation of new DNA polymerase activities i.e. reverse transcription is achievable through screening of small libraries of DNA polymerase variants that are mutated in a randomized fashion. To evolve reverse transcriptase activity in a shortened form of Thermus aquaticus DNA polymerase (Klentaq) we randomized the ORF by error prone PCR (epPCR). Protein expression was conducted in 96 well plates and enzymes were screened directly after heat denaturation of host proteins and lysis without requiring further purification steps. DNA polymerase activities were monitored by SYBRgreen I mediated quantification of PCR product formation and fluorescent readout in 384-well plates. Subsequently, the active variants were tested towards their RT activity employing a natural RNA target i.e. from bacteriophage MS2. Reverse transcription PCR (RT-PCR) activities were monitored by SYBRgreen I mediated quantification of PCR product and melting curve analysis using a conventional real-time PCR-cycler in 96-well plates. Already after screening 768 active variants we identified a promising enzyme that was subsequently purified for functional assessment. We found that this mutant exhibits strikingly increased RT-PCR activity in comparison to the wild-type enzyme. Taking together, through screening of DNA polymerase libraries that contain arbitrary randomized mutants generated by epPCR we were able to evolve enzymes that exhibit RT-PCR function, a function that is imperceptible in the wild-type enzyme. As demonstrated, the identified mutants find immediate applications and provide the basis for the development of new means for single step RT-PCR technologies like pathogen RNA detection or gene expression analysis in real-time. Noteworthy, from the same library of DNA polymerase mutants a DNA polymerase variant was identified that has increased propensity to process damaged DNA substrates when compared to the wild-type enzyme. ${ }^{9}$

\section{CONCLUSION}

The herein depicted approach of rapid DNA polymerase screening $^{10}$ can further be used to tailor DNA polymerases with new phenotypes for obtaining insights into biological processes and valuable tools for biotechnological applications and needs.

\section{REFERENCES}

1. (a) Henry, A. A., Romesberg, F. E. (2005) Curr. Opin. Biotech., 16, 370-377; (b) Brakmann, S. (2005) Cell. Mol. Life Sci., 62, 2634-2646.

2. Franklin, M. C., Wang, J., Steitz, T. A. (2001) Cell, 105, 657-667.

3. Delarue, M., Poch, O., Tordo, N., Moras, D., Argos, P. (1990) Protein. Eng, 3, 461-467.

4. Summerer, D., Rudinger, N. Z., Detmer, I., Marx A. (2005) Angew. Chem. Int. Ed., 44, 4712-4715.

5. Strerath, M., Gloeckner, C., Liu, D., Schnur, A., Marx A. (2007) ChemBioChem, 8, 395-401.

6. Rudinger, N. Z., Kranaster, R., Marx A. (2007) Chem. Biol., 14, 185-194.

7. (a) Bustin, S. A., Mueller, R. (2005) Clin. Sci., 109, 365-379; (b) Sandvik, A. K., et al. (2006) Clin. Chim. Acta, 363, 157-164; (c) Mocellin, S., Rossi, C. R., Pilati, P., Nitti, D., Marincola, F. M. (2003) Trends Mol. Med., 9, 189-195.

8. Sauter, K. B. M., Marx A. (2006) Angew. Chem. Int. Ed., 45, 7633-7635.

9. Gloeckner, C., Sauter, K. B. M., Marx A. (2007) Angew. Chem. Int. Ed., 46, 3115-3117.

10. Summerer, D., Marx, A., (2002) Angew. Chem. Int. Ed., 41, 3620-3622.

*Corresponding Author. E-mail: andreas.marx@unikonstanz.de 